\title{
JustReview \\ Uma ferramenta para otimizar o processo de revisão sistemática da literatura
}

\author{
Tulio Vitor Machado Faria ${ }^{1}$, João Bernardes Junior ${ }^{1}$ \\ ${ }^{1}$ Escola de Artes, Ciências e Humanidades - Universidade de São Paulo (EACH-USP) \\ Av. Arlindo Béttio, 100 - Ermelino Matarazo - São Paulo-SP - Brazil \\ tuliofarialgmail.com, jlbernardes@usp.br
}

\begin{abstract}
Nowadays systematic reviews are often part of academic research because through it one can identify, validate and interpret all relevant literature to a particular research question, topic or phenomenon of interest with scientific rigor and in a repeatable and well documented manner. While at first it was mostly used in the health sciences, it is being used more and more in Information Systems research as well. However, some repetitive and time consuming tasks have to be done by hand because they are not yet covered by computational aid tools. In this context, we developed JustReview, a free and open-source tool that aids the process of systematic review by facilitating and automating recurring tasks, particularly the search and download of papers from online databases and the application of criteria to accept or reject these papers, as well as the generation of documentation. This paper presents JustReview and a case study of its use.
\end{abstract}

Resumo. Atualmente a revisão sistemática faz parte de muitas pesquisas acadêmicas pois através dela é possível identificar, validar e interpretar toda a literatura relevante a uma determinada pergunta de pesquisa, tópico ou fenômeno de interesse com rigor científico e com repetibilidade e documentação do processo. Enquanto a princípio foi mais usada na área da saúde, é usada mais e mais também em pesquisa em Sistemas de Informação. Entretanto, algumas tarefas repetitivas e que consomem muito tempo se realizadas manualmente ainda não são contempladas por ferramentas computacionais para auxiliar no processo. Neste contexto, foi desenvolvido o JustReview, uma ferramenta gratuita e de código aberto que auxilia o processo da revisão sistemática da literatura através da automatização e facilitação de tarefas recorrentes nessa tarefa, particularmente a busca e download de artigos de bases de dados online e a aplicação de critérios para aceitar ou rejeitar esses artigos, assim como a geração de documentação. Esse artigo apresenta o JustReview e um estudo de caso de seu uso.

\section{Introdução}

A revisão sistemática da literatura consiste em uma forma de identificar, validar e interpretar todas as pesquisas relevantes a uma determinada pergunta de pesquisa, tópico ou fenômeno de interesse [Kitchenham 2004]. Estudos individuais que contribuem para uma 
revisão são chamados de estudos primários, já a revisão em si é definida como um estudo secundário [Kitchenham 2004].

Uma revisão da literatura é um passo inicial bastante utilizado em projetos de pesquisa e desenvolvimento, principalmente pela natureza da ciência como uma atividade colaborativa, de forma que faz-se necessário mapear e conhecer desenvolvimentos prévios na área em que se deseja contribuir[de Almeida Biolchini et al. 2007]. A revisão sistemática difere-se de outras formas de revisão de literatura por tratar-se de um trabalho replicável, com rigor científico e que minimiza a ocorrência de influências pessoais e subjetivas no processo[de Almeida Biolchini et al. 2007]. Enquanto a princípio foi mais usada na área da saúde, é usada mais e mais também em pesquisa em Sistemas de Informação.

Existem muitos motivos para se fazer uma revisão sistemática da literatura, dentre eles: conhecer de forma abrangente um determinado assunto, identificar possíveis oportunidades para futuras pesquisas ou ainda para ser utilizada como base a atividades de pesquisa [Kitchenham 2004].

Uma revisão sistemática pode ser realizada através de atividades dispostas em três fases: planejamento, condução e extração dos resultados [Kitchenham 2004].

Durante a fase de planejamento são especificadas para quais questões de pesquisa pretende-se obter respostas através da revisão. Nesta etapa são definidos fundamentalmente os objetivos específicos a serem alcançados pela mesma [Kitchenham 2004]. Planeja-se e define-se nesta fase também quais fontes de artigos serão utilizadas, como será realizada a busca nestas fontes e quais critérios serão utilizados para incluir ou excluir um estudo de acordo com sua relevância [Kitchenham 2004].

$\mathrm{Na}$ fase de condução os artigos serão encontrados e classificados como relevantes à pesquisa caso passem por critérios de inclusão e exclusão, em um primeiro momento a partir da leitura dos respectivos resumos e, posteriormente, com a leitura completa dos mesmos.

Neste contexto, durante a realização de uma revisão sistemática, notou-se uma grande dificuldade em estabelecer a priori os itens do planejamento. Isso ocorreu porque era preciso aprimorar os critérios e strings de busca durante a condução da mesma, conforme mais conhecimento sobre o tema e sobre a própria revisão ia sendo obtido, gerando grande retrabalho. Embora esse problema pudesse ser reduzido com uma revisão exploratória mais extensiva antes do planejamento, dificilmente pode ser eliminado por completo, motivo pelo qual diversos autores insistem que a revisão sistemática é um processo interativo. Assim sendo, foi proposta e desenvolvida a ferramenta livre e de código aberto batizada como JustReview, que tem como objetivo auxiliar no planejamento e condução da revisão sistemática reduzindo o trabalho manual e aumentando a produtividade.

\section{Trabalhos relacionados}

Há ferramentas no mercado que auxiliam tanto no processo de revisão sistemática quando no suporte ao gerenciamento de bibliografia. Nesta seção, serão descritas sucintamente algumas destas ferramentas. 
A ferramenta Zotero ${ }^{1}$ é essencialmente um gerenciador de bibliografia. É uma ferramenta instalada como extensão do Mozilla Firefox, porém possui uma interface web integrada, permitindo o compartilhamento dos documentos. Além disso possui aplicativos gratuitos para tablets e smartphones, como o ZotPad ${ }^{2}$, PaperShip ${ }^{3}$ e BibUp ${ }^{4}$ para iOS e Zandy ${ }^{5}$ para Android. Os recursos de adicionar rótulos, notas, extrair metadados do documento e exportar o BibTex são bastante uteis para o trabalho de revisão de bibliografia. A ferramenta Endnote ${ }^{6}$ também é uma ferramenta de gerenciamento de bibliografia comercial. Ela possui uma versão desktop e uma versão online. Permite a exportação do BibTex de artigos gerenciados pela ferramenta. Além do Zotero e do Endnote existem diversas alternativas de gerenciadores de bibliografia que podem auxiliar no processo de revisão da literatura, sistemática ou não, mas que, como será visto, não apresentam as mesmas funcionalidades que o JustReview.

A ferramenta StArt ${ }^{7}$ auxilia no planejamento, condução e execução de revisões sistemáticas. As tarefas mais custosas, no entanto, como buscar os dados bibliográficos, resumos e artigos completos ainda são realizadas de forma manual. Além disso, é uma ferramenta concebida para ser executada offline, dificultando o compartilhamento e cooperação.

A ferramenta SLR Tool ${ }^{8}$ é uma ferramenta web gratuita de revisão sistemática que permite cadastrar todos os dados da revisão sistemática. No momento de inserir os artigos e materiais encontrados, o processo é realizado manualmente. O processo de classificação exige que o pesquisador entre em artigo por artigo e rotule o mesmo como incluído ou excluído, sendo um processo bastante trabalhoso. A característica mais marcando do SLR Tool é seu suporte a multiusuários, permitindo mais de um pesquisador trabalhar em uma mesma pesquisa.

A ferramenta DestillerSR ${ }^{9}$ é uma ferramenta web comercial para a realização de revisões sistemáticas. A ferramenta permite o gerenciamento básico das informações pertinentes a revisão sistemática. Possui algumas ferramentas que facilitam o filtro em textos inseridos manualmente no sistema. Porém, seu alto custo pode ser um impeditivo em pesquisas com restrições orçamentárias.

Em todas as ferramentas encontradas específicas à revisão sistemática, não existe a possibilidade de automatizar a busca pelos dados bibliográficos, resumos e artigos em bases online a partir de uma string de busca específica. Também não há uma interface amigável web compatível com tablets e smartphones para classificação dos mesmos para uma revisão sistemática, como é proposto na ferramenta JustReview. Já as ferramentas Zotero, Endnote e similares possuem uma finalidade diferente do JustReview, atuando principalmente como gerenciadores de bibliografia.

\footnotetext{
${ }^{1}$ http://zotero.org

${ }^{2} \mathrm{http}: / / \mathrm{www} \cdot z o t p a d . c o m$

${ }^{3}$ http://www.papershipapp.com

${ }^{4}$ http://elearning.unifr.ch/bibup/tuto/

${ }^{5}$ https://play.google.com/store/apps/details?id=com.gimranov.zandy.app

${ }^{6} \mathrm{http}: / /$ endnote.com/

${ }^{7}$ http://lapes.dc.ufscar.br/tools/start_tool

${ }^{8}$ http://www.slrtool.org/

${ }^{9} \mathrm{http}: / /$ systematic-review.net/
} 


\section{A ferramenta JustReview}

A ferramenta JustReview foi desenvolvida com o objetivo principal de aumentar a produtividade em trabalhos de revisão sistemática da literatura. A motivação de desenvolvimento veio da necessidade de realizar a busca dos artigos em bases de dados inúmeras vezes até que elementos do planejamento como a string de busca exata e os critérios de inclusão e exclusão convergissem durante o processo e pela quantidade grande de artigos retornados, o que, caso fosse feito de forma manual, demandaria uma grande quantidade de tempo com trabalho repetitivo e manual.

$\mathrm{Na}$ versão atual do JustReview, pode-se citar como principais vantagens de seu uso: a grande economia de tempo para fazer o download dos artigos selecionados nas bases de dados a partir de uma string de busca; uma economia de tempo adicional com o uso de uma interface ágil listando todos os artigos obtidos em cada base, seus resumos e os critérios de inclusão e exclusão, facilitando e automatizando a seleção dos artigos e a geração dos relatórios de condução da revisão, que mostram esses dados; a redução de ocorrências de erro humano nas etapas citadas anteriormente graças à automatização das tarefas.

Um dos requisitos mais importantes para o JustReview e uma das principais lacunas encontradas em trabalhos semelhantes foi justamente essa obtenção automática dos artigos, resumos e dados bibliográficos nas bases de dados a partir de strings de busca, visto que não há uma padronização de como as bases fornecem essas informações. Para lidar com esse problema, o sistema desenvolvido cria um formato único para esses dados e isola a obtenção de dados das bases em uma camada inferior que, com scripts específicos para cada base de dados, converte os dados para o formato único, que pode então ser usado consistentemente nas camadas superiores do sistema. O JustReview também especifica como deve ser a construção desses scripts, para facilitar o uso do sistema com novas bases de dados. Atualmente o sistema tem scripts para busca nas bases ACM Digital Library, IEEE Xplore, Scopus e RBIE (Revista Brasileira de Informática na Educação).

Nas subseções seguintes são descritas a arquitetura e as tecnologias utilizadas no desenvolvimento da ferramenta JustReview, bem como suas funcionalidades.

\subsection{Arquitetura}

A ferramenta JustReview foi dividida em duas partes: JustReview Web e JustReview Bot. O módulo Web é responsável por gerenciar e organizar todos os dados das revisões sistemáticas. Já o módulo Bot é responsável por realizar as tarefas de busca de dados bibliográficos e resumos (metadados) e dos artigos completos. Na Figura 1 é mostrado a arquitetura definida, evidenciando os dois módulos e como eles se comunicam.

\subsubsection{JustReview Web}

O módulo JustReview Web é o módulo responsável pelo gerenciamento de todos os dados da revisão bibliográfica, bem como permitir classificar os artigos como excluídos ou incluídos na revisão. A decisão de fazer deste módulo uma aplicação web deu-se para permitir que a revisão sistemática seja passível de condução por mais de um pesquisador em colaboração, em uma grande gama de dispositivos e que os dados da mesma continuassem 


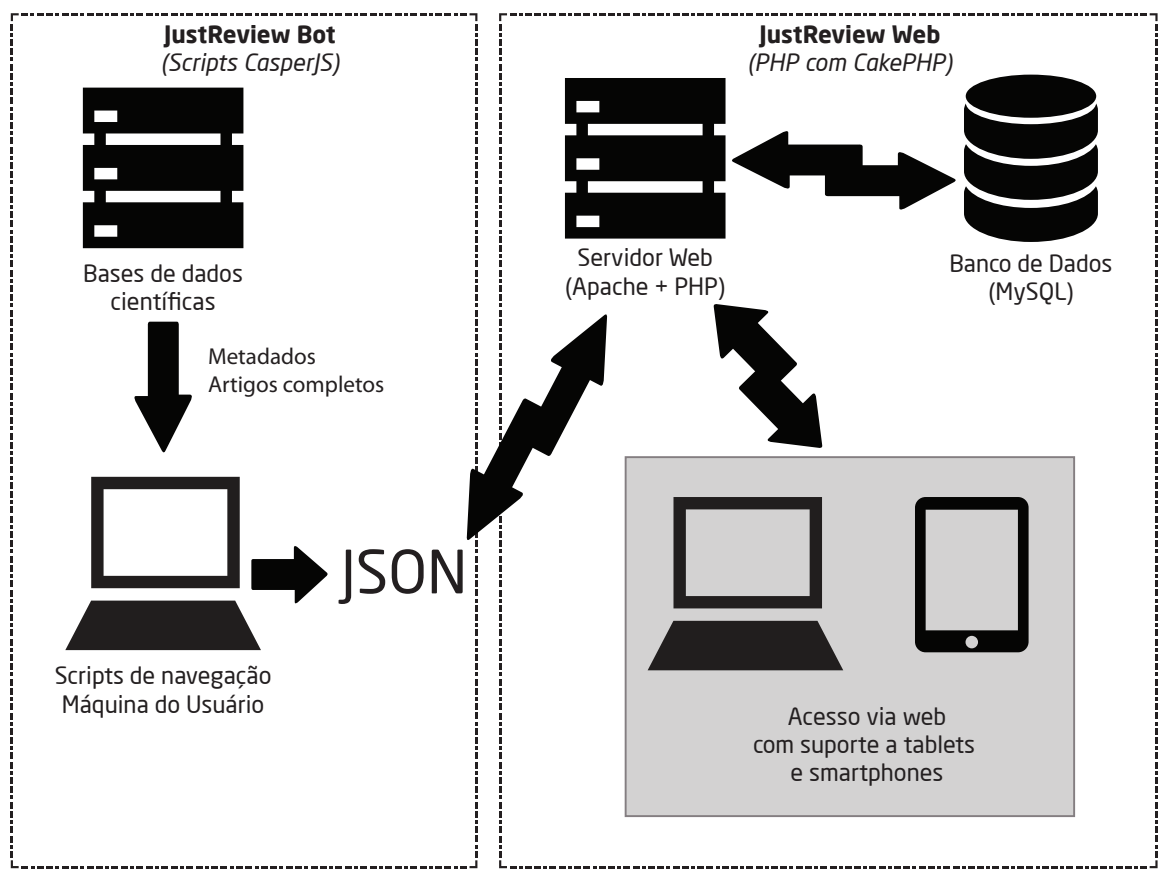

Figura 1. Arquitetura do JustReview

centralizados. Visto que revisões sistemáticas deveriam sempre ser conduzidas por equipes com mais de um pesquisador [Kitchenham 2004], considera-se esse um importante requisito.

O desenvolvimento em plataforma web foi feito utilizando a linguagem PHP em conjunto com o framework MVC CakePHP ${ }^{10}$. A adoção deste framework se deu com o intuito de facilitar que novas funcionalidades sejam adicionadas por qualquer interessado, uma vez que, conhecendo-se o framework através de um vasto material disponível online, outros desenvolvedores poderão contribuir para o projeto. Além disso, um dos autores faz uso do CakePHP a mais de 5 anos e sua familiaridade com o mesmo foi um fator importante em sua escolha.

A interface foi construída em HTML, CSS e JavaScript de forma responsiva, o que permite que uma mesma interface gráfica adeque-se automaticamente a dispositivos com telas de diferentes tamanhos e formatos, tornando o JustReview Web também compatível com tablets e smartphones. Na construção da interface foi utilizado como base o framework Bootstrap ${ }^{11}$. A Figura 2 mostra o JustReview Web sendo visualizado em dois dispositivos distintos em posição paisagem e retrato e como sua interface gráfica ajusta-se a cada um.

\subsubsection{JustReview Bot}

O módulo JustReview Bot é formado por um conjunto de scripts escritos em JavaScript que atuam como um navegador sem interface gráfica. Os scripts foram desenvolvidos

\footnotetext{
${ }^{10} \mathrm{http}: / /$ cakephp.org

${ }^{11} \mathrm{http}: / /$ getbootstrap.com
} 

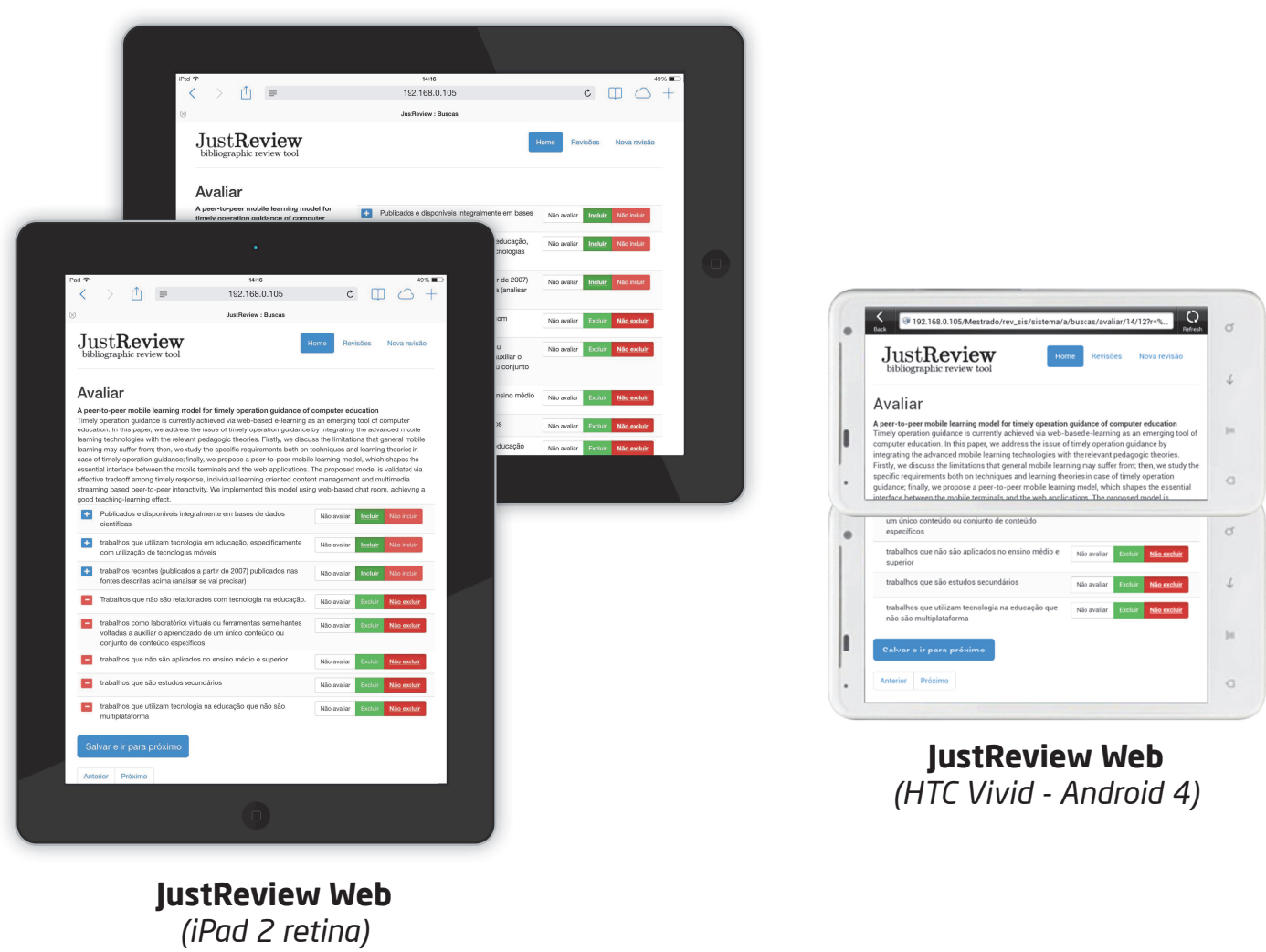

Figura 2. Visualização do JustReview Web no iPad e HTC Vivid com layout responsivo

para serem executados no CasperJS ${ }^{12}$ que utiliza o engine web WebKit (mesmo engine utilizado por navegadores como Google Chrome e Safari).

A decisão de construir o JustReview Bot independente do JustReview Web deuse com o propósito de separar as tarefas mais custosas em tempo e que consomem mais recursos computacionais da máquina do usuário em um módulo a parte. Esta arquitetura permite também o acesso a artigos em bases de dados científicas restritas, que só podem ser acessadas a partir de determinadas subredes, como por exemplo a rede de uma universidade, pois o JustReview Bot utiliza a mesma conexão com a internet do usuário.

As tarefas realizadas pelo JustReview Bot resume-se basicamente em salvar os metadados dos artigos encontrados por uma string de busca em uma base de dados ou salvar o artigo completo uma vez que ele seja classificado para leitura completa.

Durante sua execução, o JustReview Bot simula a navegação feita por um usuário, inclusive através de clicks de mouse. Sendo assim, é possível estender os scripts para suportar outras bases, inclusive bases que necessitem de autenticação.

A comunicação entre o JustReview Web e Bot é realizada através da troca de arquivos em formato JSON. Este formato foi escolhido por ser gerado e lido pelo JavaScript e PHP de forma transparente, simplificando o processo de comunicação entre eles.

\footnotetext{
${ }^{12} \mathrm{http}: / /$ casperjs.org
} 


\subsection{Projeto conceitual do banco de dados}

O banco de dados foi projetado para armazenar todas as informações gerenciadas da revisão sistemática, bem como dados de controle de acesso. Inicialmente o banco de dados possui 7 entidades, conforme apresentado na Figura 3.

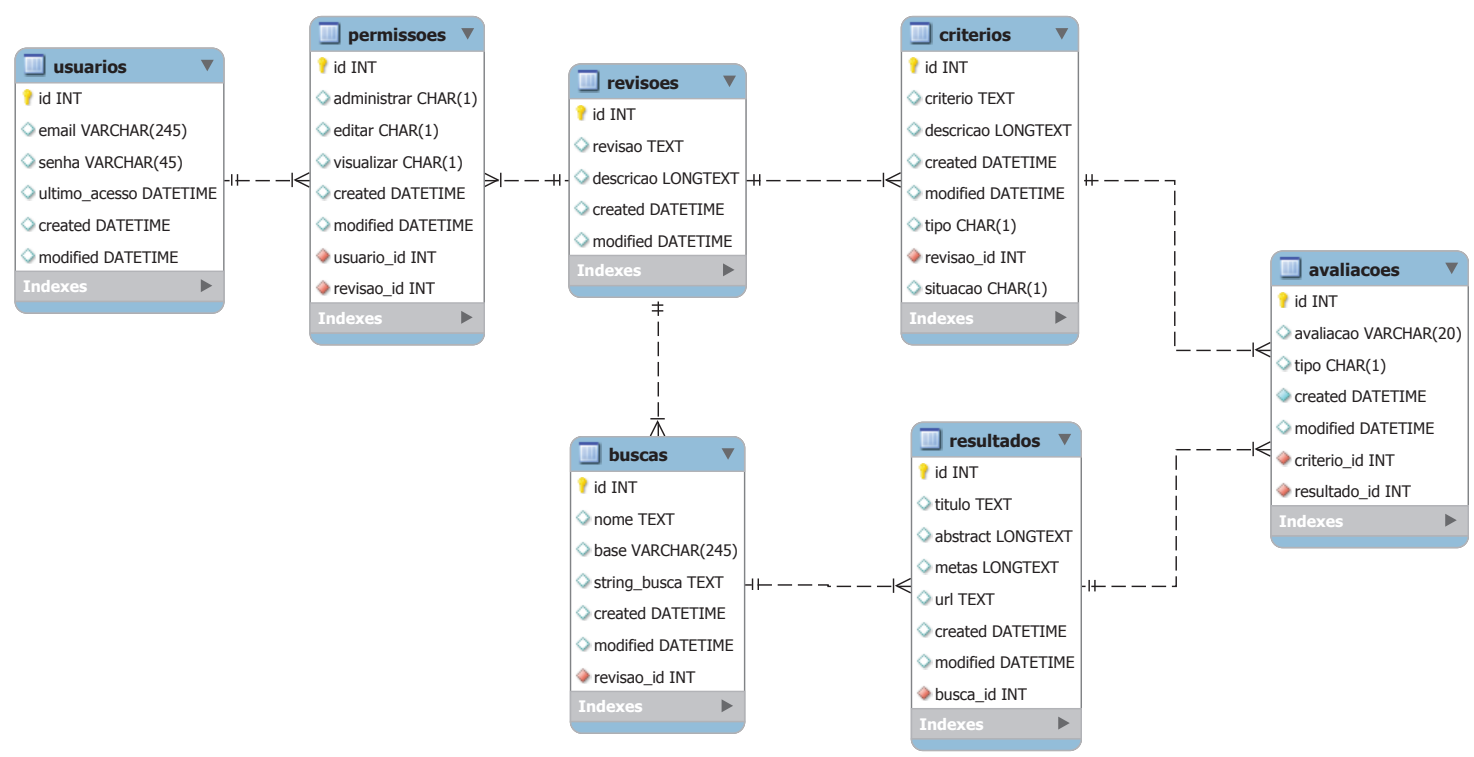

Figura 3. Modelo lógico do banco de dados

A entidade usuarios representa cada usuário do sistema, sendo considerado o cenário onde o JustReview seria instalado em produção em um servidor publicamente acessível. Cada usuário pode relacionar-se com a entidade revisoes através da entidade permissoes, que também é responsável por indicar quais operações determinado usuário pode realizar em uma revisão sistemática.

Já a entidade buscas armazena cada busca realizada pelos scripts, qual a string de busca utilizada e em qual base científica. A entidade buscas é relacionada com a entidade resultados que consiste nos registros de metadados dos artigos encontrados.

A entidade criterios armazena os critérios de inclusão e exclusão relacionados a uma revisão, e a tabela avaliacoes persiste a avaliação de um critério em relação a um resultado, onde o mesmo pode ser considerado incluído ou excluído de acordo com um dado critério.

\subsection{Funcionalidades}

A seguir são descritas em mais detalhe as principais funcionalidades do JustReview.

\subsubsection{Gerenciamento das revisões}

O JustReview Web permite, de uma forma simples e intuitiva, gerir passos trabalhosos de uma revisão sistemática. O gerenciamento de revisões permite que várias revisões sejam conduzidas e gerenciadas, além de manter um histórico permitindo que no futuro a mesma revisão seja atualizada com novos artigos que possam estar disponíveis, reduzindo o retrabalho. A Figura 4 mostra a tela com a listagem de revisões cadastradas. 


\section{JustReview}

bibliographic review tool

\section{Revisão}

Revisão de metodologia da pesquisa científica

Buscar artigos que mostrem tecnologias aplicadas a educação.
Ações

Critérios | Buscas | Latex | Duplicados | Editar

(C) Desenvolvido por: Tulio Faria EACH 2014

Figura 4. Tela com a lista de revisões já criadas

\subsubsection{Realizando uma busca com o JustReview Bot}

A primeira tarefa realizada pelo JustReview Bot em uma revisão sistemática é a busca de metadados dos artigos em uma dada base de dados. Para realizar esta tarefa de busca na RBIE - Revista Brasileira de Informática na Educação, depois de instalado o CasperJS no sistema, basta executar na linha de comando:

casperjs busca-rbie.js "string de busca"

Após a execução, que pode demorar alguns minutos, será gerado um arquivo no mesmo diretório com o nome: rbie-data-ANO-MÊS-DIA.json. Este arquivo será utilizado no momento do cadastro da busca conforme descrito em 3.3.3.

\subsubsection{Gerenciamento de buscas}

As buscas dentro do JustReview Web contém informações sobre como os artigos serão buscados em uma base de dados, e principalmente, qual a base de dados e qual a string de busca utilizada. No cadastro de uma nova busca, além destas informações é realizado o envio de um arquivo JSON gerado pelo JustReview Bot. Este arquivo já possui os metadados coletados pelo Bot e já possibilita que os artigos comecem a ser avaliados e classificados.

\subsubsection{Classificação dos artigos}

A classificação dos artigos é uma das tarefas mais importantes e também uma das mais trabalhosas de uma revisão sistemática. Durante a classificação, o JustReview Web exibe o título e abstract, e os confronta com os critérios de inclusão e exclusão, e permite ao usuário escolher se o artigo foi incluído ou excluído por cada critério. Um artigo é considerado como relevante ou aprovado se foi incluído por pelo menos um critério de inclusão, e considerado excluído ou reprovado se for excluído por pelo menos um critério de exclusão. Os textos para os critérios de inclusão e exclusão são cadastrados pelos usuários de forma simples na interface de gerenciamento da revisão. A Figura 5 mostra a tela de classificação no JustReview Web. 


\section{JustReview}

\section{Avaliar}

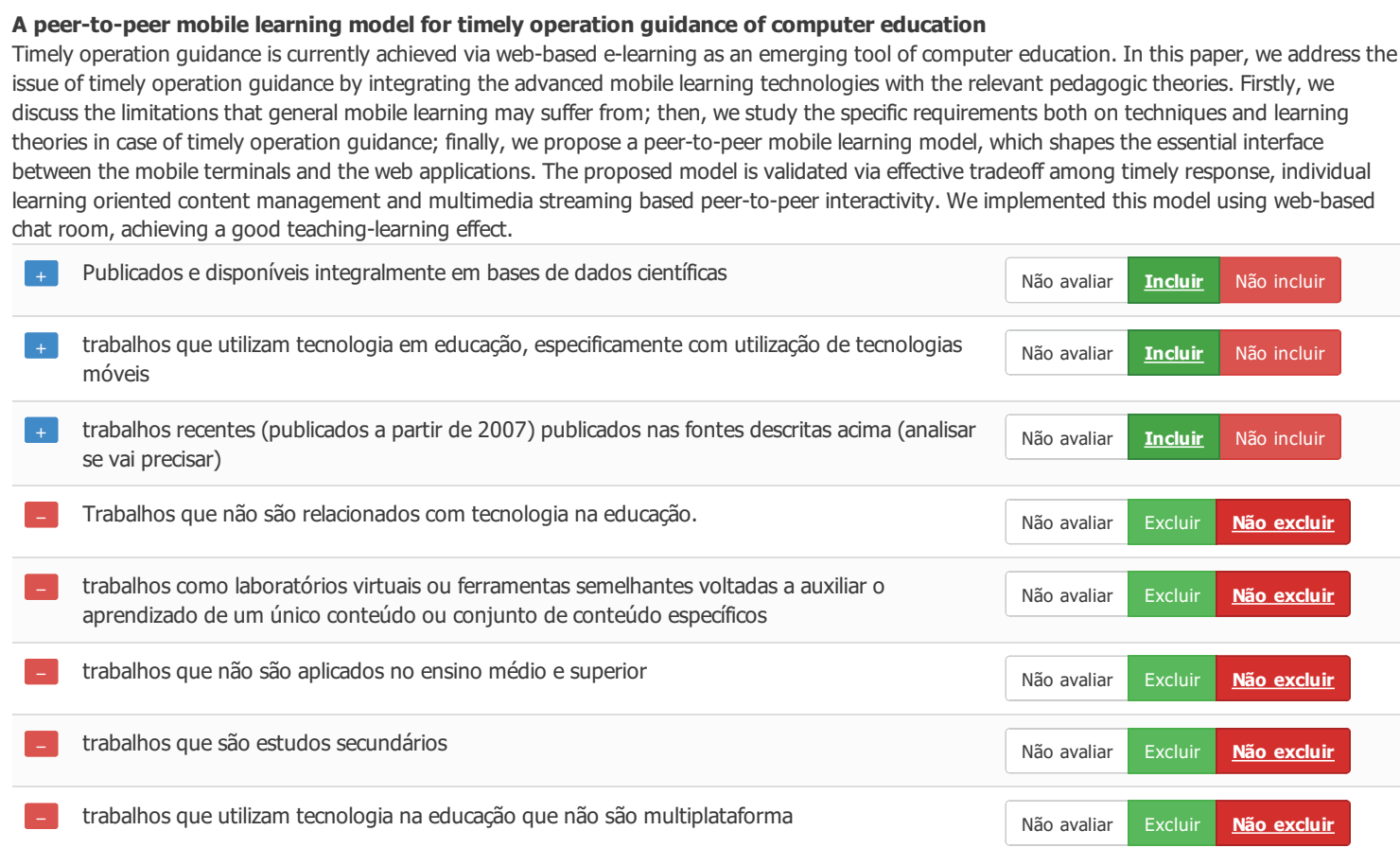

\section{Salvar e ir para próximo}

Anterior Próximo

(c) Desenvolvido por: Tulio Faria EACH 2014

Figura 5. Tela de avaliação de artigos do JustReview Web

Na listagem das buscas realizadas há a possibilidade de visualizar um sumário de quantos artigos já foram classificados, e também quais foram aprovados e a possibilidade de reclassificar o mesmo.

\subsubsection{Geração do relatório de condução em LaTeX}

O relatório de condução da revisão sistemática pode ser gerado em LaTeX após a classificação de todos os artigos de todas as buscas. Esse relatório mostra todos os dados da revisão como a lista de critérios, em quais bases foi realizado a busca de artigos, qual string de busca utilizada, a lista de artigos, com quais critérios ele foi incluído ou excluído e o resultado (incluído ou excluído). A Figura 6 mostra a primeira página do protocolo proveniente do LaTeX gerado pelo JustReview Web. 
1 Critérios de inclusão e exclusão

\subsection{Inclusão}

\begin{tabular}{|l|l|}
\hline a & $\begin{array}{l}\text { publicados e disponíveis integralmente em bases de dados } \\
\text { científicas }\end{array}$ \\
\hline b & $\begin{array}{l}\text { trabalhos que utilizam tecnologia em educação, especificamente } \\
\text { com utilização de tecnologias móveis }\end{array}$ \\
\hline c & $\begin{array}{l}\text { trabalhos recentes (publicados a partir de 2007) publicados nas } \\
\text { fontes descritas acima (analisar se vai precisar) }\end{array}$ \\
\hline
\end{tabular}

\subsection{Exclusão}

\begin{tabular}{|l|l|}
\hline $\mathrm{d}$ & Trabalhos que não são relacionados com tecnologia na educação. \\
\hline $\mathrm{e}$ & $\begin{array}{l}\text { trabalhos como laboratórios virtuais ou ferramentas semelhantes } \\
\text { voltadas a auxiliar o aprendizado de um único conteúdo ou con- } \\
\text { junto de conteúdo específicos }\end{array}$ \\
\hline $\mathrm{f}$ & trabalhos que não são aplicados no ensino médio e superior \\
\hline $\mathrm{g}$ & trabalhos que são estudos secundários \\
\hline $\mathrm{h}$ & $\begin{array}{l}\text { trabalhos que utilizam tecnologia na educação que não são multi- } \\
\text { plataforma }\end{array}$ \\
\hline
\end{tabular}

\section{Condução da revisão sistemática}

\subsection{IEEE}

Fonte: IEEE

String utilizada: ((teach OR learn OR education) AND ("computer assisted" OR "computer aided" OR "computer supported") AND mobile AND (web OR internet))

\begin{tabular}{|l|l|l|l|}
\hline Artigo & $\begin{array}{l}\text { Critérios de } \\
\text { inclusão }\end{array}$ & $\begin{array}{l}\text { Critérios de } \\
\text { exclusão }\end{array}$ & Resultado \\
\hline $\begin{array}{l}\text { Web Robot Learning Powered by Bluetooth Com- } \\
\text { munication System }\end{array}$ & a,c & d,e,f,h & EXCLUÍDO \\
\hline
\end{tabular}

Figura 6. Primeira página do protocolo de condução da revisão sistemática

\subsubsection{Obtendo os artigos completos com o JustReview Bot}

A obtenção dos artigos completos das bases de dados em formato PDF é realizado pelo JustReview Bot utilizando o arquivo JSON gerado pelo JustReview Web. Este arquivo contém os dados necessários para obter os artigos classificados como incluídos na revisão. Por exemplo, para obter os artigos completos da RBIE bastaria executar a seguinte linha de comando:

casperjs pdf-rbie.js artigos-rbie.json subDiretórioDestino

Onde $p d f$-rbie.js é o script do JustReview Bot que irá ser executado pelo CasperJS, artigos-rbie.json é o arquivo JSON gerado pelo JustReview Web com os artigos a serem baixados, e subDiretoórioDestino é o diretório onde deverão ser salvos os artigos completos. Após a execução, que pode durar alguns minutos, os artigos completos estarão todos neste diretório. 


\section{Estudo de caso}

O JustReview foi utilizado durante a condução da revisão sistemática realizada por alunos de mestrado durante a disciplina de Metodologia da Pesquisa Científica no curso de mestrado em Sistemas de Informação da EACH-USP. A utilização do sistema foi de grande auxílio para a realização da mesma, principalmente pela dificuldade em definir e estabelecer as bases de dados e strings de busca visto que, na maioria dos casos, os alunos tinham relativamente pouca experiência com os temas de suas revisões e tiveram pouco tempo para executar uma revisão exploratória.

Nesta situação, o JustReview foi utilizado para fazer várias buscas em várias bases de dados até que fosse possível estabilizar e definir o planejamento da revisão. Caso o mesmo processo tivesse sido feito de forma manual ou sem auxílio da ferramenta, dificilmente haveria tempo hábil para a realização do mesmo. Após a convergência do planejamento da revisão, a busca realizada em uma única revisão (sobre uso de sistemas de informação na educação), que serve como exemplo nos próximos parágrafos, resultou em 751 artigos provenientes da IEEE Xplore, ACM Digital Library e RBIE. Os artigos encontrados foram posteriormente classificados.

$\mathrm{Na}$ etapa de classificação dos artigos dessa revisão, o JustReview foi intensamente utilizado, pois foi possível realizar esta tarefa em diferentes locais usando diferentes dispositivos como o tablet ou o computador. Além de permitir revisar uma classificação de um artigo, caso tenha ocorrido algum erro no mesmo. Nesse estudo de caso, houve quatro ocasiões em que uma classificação foi considerada incorreta e um artigo precisou ser reclassificado. A classificação para esta revisão sistemática resultou em 91 artigos classificados como incluídos e que precisaram ser obtidos por completo das bases de dados (pois até esse ponto somente os dados bibliográficos e resumos haviam sido obtidos).

A etapa de obtenção dos artigos completos foi realizada pelo JustReview Bot, e os 91 artigos completos foram obtidos. O processo foi realizado em aproximadamente 20 minutos, tempo bastante inferior ao necessário caso essa tarefa tivesse sido feita de forma manual.

\section{Trabalhos futuros}

O JustReview possui vários pontos que podem ser aprimorados no futuro. O primeiro deles é consolidar o projeto ainda mais como uma ferramenta livre e de código aberto, atraindo mais colaboradores para ampliar suas funcionalidades, principalmente a inclusão de novas bases para o JustReview Bot, e que elas também possam englobar e facilitar ainda mais tarefas realizadas em revisões sistemáticas. Agora que o projeto está sendo explorado por mais usuários (por exemplo em uma disciplina do Instituto de Matemática e Estatística da USP) e em vias de ser disponibilizado no sistema Nuvem USP, espera-se que haja mais colaborações para o sistema, provenientes de uma comunidade maior de usuários e possíveis desenvolvedores.

Outro importante aspecto a ser explorado no futuro no JustReview é ampliar seu suporte a revisão realizada por vários usuários. Além disso, obter uma versão nativa para Android e iOS que permita a sincronização com a versão pública na web e que permita a classificação dos artigos mesmo sem acesso à internet.

Por fim, pretende-se realizar estudos de usabilidade do sistema e aperfeiçoar o 
atual protótipo da interface com o usuário com base nesses estudos.

\section{Conclusão}

Este artigo apresentou a ferramenta JustReview que tem por objetivo auxiliar pesquisadores no processo de revisar sistematicamente a literatura. Os resultados obtidos com a ferramenta foram bastante satisfatórios, uma vez que ela reduziu muito o tempo de realização de tarefas repetitivas, além de facilitar o processo quando há um grande número de artigos envolvidos. O uso do sistema colaborou principalmente com a economia de tempo e redução de erros nas tarefas de obtenção e seleção dos artigos e geração dos relatórios de condução, automatizando essas tarefas.

As tecnologias definidas para o desenvolvimento do JustReview também se mostraram eficientes durante seu uso, obtendo tempos reduzidos de resposta, além de serem de uso comum para que novos colaboradores possam contribuir com o projeto.

O trabalho também atraiu a atenção de outros pesquisadores, que também fizeram uso e já propuseram novas funcionalidades e se ofereceram para ser colaboradores, um ótimo resultado para um projeto livre e de código aberto. Além disso, espera-se uma maior adoção da revisão sistemática da literatura em trabalhos científicos através da otimização e organização do mesmo através da ferramenta JustReview.

\section{Referências}

de Almeida Biolchini, J. C., Mian, P. G., Natali, A. C. C., Conte, T. U., and Travassos, G. H. (2007). Scientific research ontology to support systematic review in software engineering. Advanced Engineering Informatics, 21(2):133-151.

Kitchenham, B. (2004). Procedures for performing systematic reviews. Keele, UK, Keele University, 33:2004. 